\title{
Cosmological Energy
}

Looking at the problems of energy in the Universe is a special way of looking at the astrophysical problems. The ways in which energy is distributed or stored, the mechanisms by which energy is converted are fundamental not only to astrophysics but perhaps to physics.

Let us consider first the ways in which energy is distributed. Gravitation permeates all the Universe. Gravitational energy is not localized, but is a property of any set of gravitational bodies. Einstein's equation of general relativity can be considered as an equation of conservation of energy. It is not difficult to convert gravitational energy: a gravitating system always has the tendency to collapse. Gravitational energy is then changed into kinetic energy. In the case of random motion, it is changed into heat. Conversely, kinetic energy can be lost and converted into gravitational energy.

The " big bang" or, in other words, the expansion of the Universe, can be described as the continuous conversion of kinetic energy into gravitational energy for the whole Universe at least in Newtonian cosmology. To establish the proof of the big bang is in some ways as important as proving or controverting the law of conservation of energy.
Going backwards in the history of the Universe, we meet epochs with different processes of energy conversion (Table I). Beyond $t=10^{-4} \mathrm{~s}$, due to difficulties with the thermodynamics at high energy densities, it is difficult to ensure that the present theories are more than speculation. However, the difficulties of understanding the early stages of the expanding universe can be tackled and perhaps mastered. Studies of nucleosynthesis and the formation of galaxies are most fascinating. The possibility even exists that the Universe has kept black holes produced in the early stages. Conversion of gravitational energy into light and other observable effects take place in the stars. The chemical Rayleigh-Jeans time scale simply expresses the time during which a star can shine on its gravitational energy. Things are quite clear as long as the stellar matter is non relativistic: a star is a bound system with negative energy and a negative specific heat. However, when the velocity of particles approaches the velocity of light, the binding energy of the star can vanish as in white dwarfs with a certain critical value of the mass, to which corresponds a certain value of the radius: $1.34 \mathrm{Mo}$ and about $10^{3} \mathrm{~km}$.

A star can have a negative energy and nevertheless be unstable: this happens when the compressibility of the stellar matter becomes less than $4 / 3$. Such a situation can arise when some reaction takes place, like the production of electron-positron pairs out of the radiation field, or the dissociation of iron.

Observations show many ways in which gravitational energy is liberated. Supernovae and pulsars are well associated, and pulsars are almost certainly neutron stars, with a large binding energy. However, as far as we understand the way in which the pre-supernova stage is reached, it would be fair to say that gravitational energy raises the internal energy up to the point where explosive thermonuclear reactions can take place. Formation of nuclei with a large binding energy like ${ }^{12} \mathrm{C},{ }^{16} \mathrm{O},{ }^{20} \mathrm{Ne},{ }^{24} \mathrm{Mg}$ result from the history of the star and is the price which the star has paid for shining ; ${ }^{12} \mathrm{C}$ and ${ }^{16} \mathrm{O}$ when explosively producing nuclei of great binding energy, become responsible for the supernova outburst.

Supernova outburst, or possibly non explosive collapse leads to the formation of a neutron star. In the compact variable $\mathrm{X}$-ray sources, the neutron star is companion of a larger bright star. Such a system has been formed in the course of stellar evolution, and the matter shed by the primary on to the neutron star, after a number of events, produces the $\mathrm{X}$-rays. Variability is due to the rotation of the neutron star and to the eclipse by the primary (Her X1, Cen X1).

The possibility that evolution, instead of leading to a neutron star, companion of a normal star, generates a black hole is not excluded. Cyg X1 may be the product of such an evolution, the $\mathrm{X}$-rays this time being produced by accretion on the black hole.

What can we say about the other forms of energy conversion, which can take place with black holes? Merging of non-rotating or rotating black holes, interaction of E-M radiation with a black hole lead to interesting results : for example, two rotating black holes rotating in opposite directions with the maximum angular momentum can merge into a nonrotating black hole, energy being converted with a $50 \%$ efficiency. It is however liberated in gravitational waves of little physical impact. 
However, when introducing quantum effects, a black hole can emit particles, created by vacuum fluctuations near the event horizon. Being a body of negative specific heat, a black hole which cools sees its equivalent temperature increasing or its mass decreasing. Possibly, black holes of any mass might have been formed in the early Universe. According to Hawking, no black hole of mass less than $10^{15} \mathrm{~g}$ should be left in the Universe. All black holes with smaller mass have "exploded " long ago.

The maximum mass (due to General Relativity effects) of neutron stars is also the minimum mass for present formation of black holes. This depends very much on the physics of dense matter at densities larger than $10^{15} \mathrm{~g} / \mathrm{cm}^{3}$, or at mutual distances of baryons less than 1 Fermi. Similar to the problem of nuclear matter, this is a very difficult many body problem. Maximum masses of neutron stars range presently from 1.6 to 2.5 solar masses. The invisible companion of Cyg $\mathrm{X} 1$, with a present estimate of 6 Mo might very well be a black hole.

At the other end of the spectrum, production of radio waves with a high efficiency is a characteristic of several astronomical objects : radio galaxies, pulsars.

All problems of interpretation rely on the observations. In the case of radio-galaxies, it appears clearly now that matter is recurrently ejected and emitted by synchrotron radiation. However, the discovery in some frequency ranges of spikes of radiation with a duration of $10^{-6} \mathrm{~s}$ and an effective temperature of $10^{31} \mathrm{~K}$ suggests strongly a maser-like mechanism of emission for which no model is yet available. The only certainty is that the emission region has the size of about $1 \mathrm{~km}$, much less than the size of the star, which is perhaps 10 or $20 \mathrm{~km}$ in radius.

Nuclei of galaxies are quite remarkable sources of radiation, undoubtedly parent to radio galaxies. No model seems yet available to explain completely the energy source, the variability, the spectrum, the compactness. There is no lack of imagination: the nucleus of an active galaxy has been thought to be a compact cluster of pulsars, a super-massive rotating magnetic body, an accreting black hole, a system of matter and antimatter. None of those models is able to explain all the major properties of the nuclei of galaxies.

E. S.
Journal of Magnetism and

\section{Magnetic Materials}

EDITOR:

A. J. FREEMAN - Department of Physics, Northwestern University, Evanston, Illinois 60201, U.S.A.

\section{ASSOCIATE EDITORS:}

R. S. TEBBLE - Department of Pure \& Applied Sciences, University of Salford, Salford M5 4WT, England

R. J. VARNERIN - Bell Telephone Laboratories, Murray Hill, New Jersey, 07974, U.S.A.

\section{EDITORIAL ADVISORY BOARD:}

A. Arrott (Canada), A. Blandin (France), J. I. Budnick (U.S.A.), A. M. Clogston (U.S.A.), S. Doniach (U.S.A.), S. Foner (U.S.A.), F. J. Friedlaender (U.S.A.), V. Jaccarino (U.S.A.), I. S. Jacobs (U.S.A.), H. S. Jarrett (U.S.A), C. E. Johnson (England), W. C. Koehler (U.S.A.), E. I. Kondorsky (U.S.S.R.), J. S. Kouvel (Canada), F. Luborski (U.S.A.), S. Lundqvist (Sweden), A. R. Mackintosh (Denmark), S. Methfessel (West Germany), A. R. Miedema (The Netherlands), A. Misetich (Argentina), B. Mühlschlegel (West Germany), S. Oshima (Japan), L. Pál (Hungary), A. Paoletti (Italy), R. Pauthenet (France,) G. T. Rado (U.S.A.), E. J. Samuelson (Norway), S. Shtrikman (Israel), J. C. Slonczewski (U.S.A.), R. Stevenson (Canada), E. D. Thompson (U.S.A.), E. J. Torok (U.S.A.), J. H. van Vleck (U.S.A.), S. V. Vonsovsky (U.S.S.R.), R. E. Watson (U.S.A.), E. P. Wohlfarth (England), K. Yosida (Japan).

\section{SCOPE:}

International research in the broad field of magnetism covering the whole spectrum of topics from basic science to the technology and application of magnetic materials, continues to grow at a rapid rate. This has led to the strong conviction that there is a great need for a forum for the discussion and disclosure of new and original work in this important area. The JOURNAL OF MAGNETISM AND MAGNETIC MATERIALS has been established to encourage greater interaction between the basic and applied sub-disciplines of magnetism, and to offer a natural channel for the dissemination of ideas and developments on the widest basis which cannot be achieved in any other way. In addition to the more traditional publications, timely review articles and a separate "Letters to the Editor" section will be featured; to achieve more rapid publication, galleys of Letters will not be sent to authors.

\section{INVITATION TO AUTHORS:}

The editors invite original contributions on all aspects of fundamental and applied problems in the study of magnetism and magnetic materials. Contributions should be submitted preferably to one of the editors or to a member of the Editorial Advisory Board who is familiar with the research presented. "Instructions to Authors" are available from the publishers. There will be no publication charge, and 25 reprints will be provided free of charge, with additional reprints available from the publisher at their normal prices.

\section{SUBSCRIPTION INFORMATION:}

The first issue of the JOURNAL OF MAGNETISM AND MAGNETIC MATERIALS is scheduled for October 1, 1975. There will be 4 issues per volume and it is anticipated that 3 volumes will be printed per year.

The subscription price for Volume 1 is US $\$ 52.95 / \mathrm{Dfl} .132 .00$ including postage. Specimen copies are available upon request.

Journals are automatically sent by air to the U.S.A. and Canada at no extra cost, and to Japan, Australia and New Zealand with a small additional postage charge.

Subscriptions should be sent to the publisher

\section{North-Holland Publishing Co.}

P.O. Box 211, Amsterdam, The Netherlands

puces ate subject to change without prior notice 